\title{
Optimal laser control of double quantum dots
}

\author{
E. Räsänen,,2,* A. Castro, ${ }^{1,2}$ J. Werschnik, ${ }^{1,2,3}$ A. Rubio, ${ }^{1,2,4}$ and E. K. U. Gross ${ }^{1,2}$ \\ ${ }^{1}$ Institut für Theoretische Physik, Freie Universität Berlin, Arnimallee 14, D-14195 Berlin, Germany \\ ${ }^{2}$ European Theoretical Spectroscopy Facility (ETSF) \\ ${ }^{3}$ JENOPTIK Laser, Optik, Systeme GmbH, Göschwitzer Strasse 25, D-07745 Jena, Germany \\ ${ }^{4}$ Departamento de Física de Materiales, Facultad de Químicas, Centro Mixto CSIC, and Donostia International Physics Center (DIPC), \\ Universidad del País Vasco, E-20018 Donostia-San Sebastián, Spain
}

(Received 16 October 2007; revised manuscript received 20 December 2007; published 27 February 2008)

Coherent single-electron control in a realistic semiconductor double quantum dot is studied theoretically. Using optimal-control theory, we show that the energy spectrum of a two-dimensional double quantum dot has a fully controllable transition line. We find that optimized picosecond laser pulses generate population transfer at significantly higher fidelities $(>0.99)$ than conventional sinusoidal pulses. Finally, we design a robust and fast charge switch driven by optimal pulses that are within reach of terahertz laser technology.

DOI: 10.1103/PhysRevB.77.085324

Double quantum dots (DQDs), i.e., coupled twodimensional (2D) electron traps, have been under recent and extensive studies both experimentally ${ }^{1-3}$ and theoretically. ${ }^{4-8}$ The main interest in DQDs arises from their potential for solid-state quantum computation that could be achieved, in principle, by rapidly switching voltages of electrostatic gates. The gates permit to tune at will the system geometry and hence the electronic properties of DQDs. Coherent manipulation of a single charge ${ }^{2}$ and coupled spins ${ }^{1}$ has already been achieved, and recently, a coherence time of $\sim 200 \mathrm{~ns}$ was obtained for a well isolated silicon DQD. ${ }^{3}$ Theoretical studies on single-electron transport inside the DQD driven by linear switches and linearly polarized continuous waves (cw's) were reported very recently. ${ }^{4}$ In the latter case, the transport is rather sensitive to possible anharmonicity of the potential and limited to uncoupled dots far apart from each other. Electron control in DQDs has been studied also using genetic algorithms ${ }^{5}$ as well as rotating-wave and resonant approximations, leading to a reduction to a three-level system. ${ }^{6}$ To the best of our knowledge, however, a general $\mathrm{N}$-level control scheme by using direct external electric fields has not been introduced for 2D-DQDs until now.

In this paper, we discuss the controllability criteria for single-electron states of DQDs by means of external laser pulses. We show that at certain interdot distances, some of the single-electron states allow full population transfer from the ground state to those states. We apply quantum optimalcontrol theory $(\mathrm{OCT})^{9}$ which yields the optimal laser pulses for predefined transitions. We obtain high occupations $(\gtrsim 99 \%)$ of the target states in a realistic DQD in a few picoseconds, which is well in the coherent regime. If the initial and final states are chosen to have full localization of the electron in one or the other dot, this scheme enables rapid and controlled transport which is not sensitive to the interdot distance or to the inevitable anharmonicities in the confining potential.

In the static 2D Hamiltonian, $\hat{H}_{0}=-\left(\partial_{x}^{2}+\partial_{y}^{2}\right) / 2+V_{\mathrm{c}}(x, y)$, the external potential describing the DQD is, in its most common form, ${ }^{8}$ given by

$$
V_{\mathrm{c}}(x, y)=\frac{\omega_{0}^{2}}{2} \min \left[\left(x-\frac{d}{2}\right)^{2}+y^{2},\left(x+\frac{d}{2}\right)^{2}+y^{2}\right],
$$

where $d$ is the distance between the potential minima and $\omega_{0}=0.5$ is the confinement strength with a typical value for DQDs. We apply the effective-mass approximation for electrons moving in GaAs and set the effective mass to $m^{*}$ $=0.067 m_{e}$ and the dielectric constant to $\kappa=12.7 \epsilon_{0}$. The energies, lengths, and times are given in effective a.u.: $\mathrm{Ha}^{*}=\left(m^{*} / \kappa^{2}\right) \mathrm{Ha} \approx 11.30 \mathrm{meV}, \quad a_{0}^{*}=\left(m^{*} / \kappa\right) a_{0} \approx 10.03 \mathrm{~nm}$, and $u_{t}^{*}=\hbar / \mathrm{Ha}^{*} \approx 58.23 \mathrm{fs}$, respectively.

In Fig. 1, we show the lowest energy levels as a function of $d$ (left panel) as well as densities of six lowest eigenstates

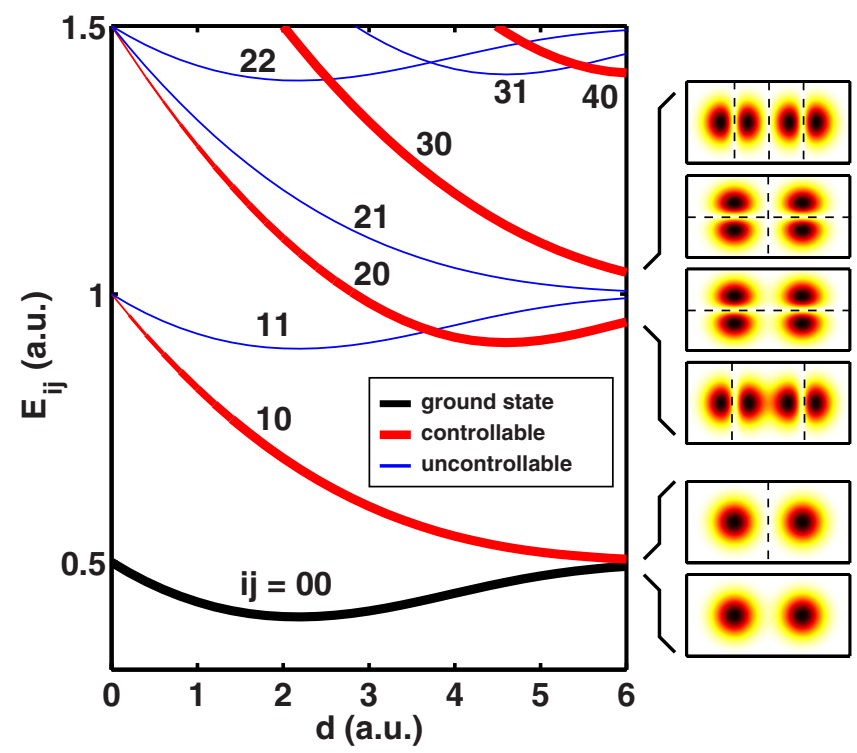

FIG. 1. (Color online) Left panel: lowest eigenenergies of a double quantum dot with $\omega_{0}=0.5$ as a function of the interdot distance. Black, red (thick), and blue (thin) curves mark the ground state, controllable states, and uncontrollable states, respectively. Right panel: densities of six lowest eigenstates at $d=5$. The dashed lines mark the nodes of the wave functions. 
at $d=5$ (right panel). The $d=0$ limit corresponds to the wellknown shell structure of a single 2D harmonic oscillator (HO), where the energy levels are $n$-fold degenerate at energies $n \omega_{0}(n=1,2, \ldots)$. Increasing $d$ leads to lifting of the degeneracies and generates crossings and avoided crossings between the energy levels. In the weak-coupling limit $d \rightarrow \infty$, the bundling of the levels at $n \omega_{0}$ is restored. In this limit, the energies are $2 n$-fold degenerate corresponding to two (uncoupled) HOs. We label the states in the DQD as $\left|\psi_{i j}\right\rangle=|i j\rangle$, where $i=0,1, \ldots$ denotes the $(i+1)$ th bundle of states at $d=0$, and $j=0,1, \ldots$ denotes the $(j+1)$ th state in each bundle. As visualized in the right panel of Fig. 1, index $i$ also corresponds to the number of nodes in the wave function. We show below that our labeling yields simple rules for controllable and uncontrollable states as a function of $d$.

It is a well-known fact in control theory that an infinitelevel single $\mathrm{HO}$ is not controllable in the dipole approximation. ${ }^{10}$ This is in contrast with the truncated $\mathrm{HO}$ which (in most cases) satisfies the controllability criteria. ${ }^{11}$ In qualitative arguments, the uncontrollability of a single $\mathrm{HO}$ $(d=0)$ in the dipole approximation stems from the equidistant single-electron level spacings. Using the above labeling of the states, the nonvanishing dipole-matrix elements $\langle i j|\hat{\mu}| k l\rangle$ between the $\mathrm{HO}$ states $(d=0)$ are

$$
\begin{aligned}
& |\langle i, j|\hat{\mu}| i+1, j+1\rangle|=\sqrt{2(j+1)}, \\
& |\langle i, j|\hat{\mu}| i+1, j\rangle|=\sqrt{2(i-j+1)},
\end{aligned}
$$

where $\hat{\mu}=-e \mathbf{r}$ is the dipole operator. Equation (2) holds for all values of $d$, and the corresponding energy-level spacings remain constant. Hence, transitions $i j \rightarrow(i \pm 1)(j \pm 1)$ remain uncontrollable. On the other hand, the elements given in the left-hand side (LHS) of Eq. (3) change as a function of $d$, as well as the level spacings. The behavior already suggests that transitions $i j \rightarrow(i \pm 1) j$ become controllable when $d$ is increased from zero. Our calculations below confirm this prediction. Starting with the ground state $|00\rangle$, the controllable transition line is then $|00\rangle \rightarrow|10\rangle \rightarrow|20\rangle \ldots$ (see the red curves in Fig. 1). In the weak-coupling limit $(d \rightarrow \infty)$, however, the LHS of Eq. (3) becomes

$$
\lim _{d \rightarrow \infty}|\langle i, j|\hat{\mu}| i+1, j\rangle|= \begin{cases}\infty & \text { if } i-j \text { is even } \\ \sqrt{2(i-j)} & \text { if } i-j \text { is odd, }\end{cases}
$$

so that only the trivial transitions between the degenerate gerade and ungerade states are possible. In the large- $d$ regime, however, breaking the interdot symmetry leads to the possibility of charge transport between the dots (see below).

Next, we apply OCT in order to find optimal laser pulses for transitions from the initial state $\left|\Phi_{\mathrm{I}}\right\rangle=|\Psi(t=0)\rangle$ to the target state $\left|\Phi_{\mathrm{F}}\right\rangle=|\Psi(t=T)\rangle$ in a fixed time interval $T$. In the OCT formalism, we maximize the overlap $\left|\left\langle\Psi(T) \mid \Phi_{\mathrm{F}}\right\rangle\right|^{2}$ while minimizing the fluence of the laser pulse. The control equations $\operatorname{are}^{12}$

$$
\begin{gathered}
i \partial_{t} \Psi(t)=\hat{H} \Psi(t), \quad \Psi(0)=\Phi_{\mathrm{I}}, \\
i \partial_{t} \chi(t)=\hat{H} \chi(t), \quad \chi(T)=\Phi_{F}\left\langle\Phi_{F} \mid \Psi(T)\right\rangle,
\end{gathered}
$$

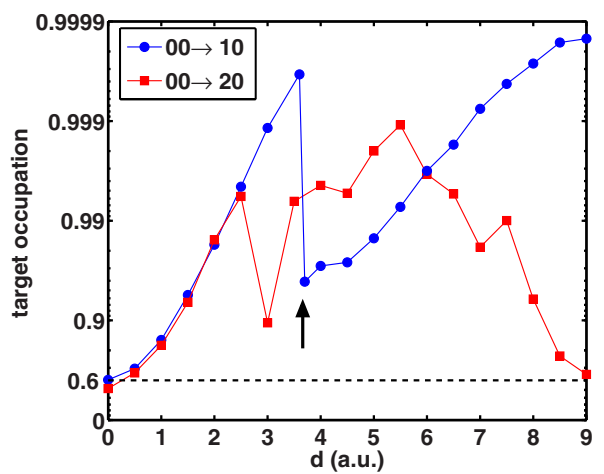

FIG. 2. (Color online) Maximum occupation (logarithmic scale) of the target state as a function of the interdot distance $d$ in transitions $|00\rangle \rightarrow|10\rangle$ with pulse length $T=50$ (circles) and $|00\rangle \rightarrow|20\rangle$ with $T=100$ (squares). The lines are guides for the eyes. The dashed line denotes the maximum target-state occupation of 0.6 for a single harmonic oscillator. The jump marked by an arrow is due to a resonance effect (see text).

$$
\boldsymbol{\epsilon}(t)=-\frac{A(t)}{\alpha} \operatorname{Im}\langle\chi(t)|\hat{\mu}| \Psi(t)\rangle,
$$

where Eq. (5) is the time-dependent Schrödinger equation with $\hat{H}=\hat{H}_{0}-\hat{\mu} \boldsymbol{\epsilon}(t)$ and $\chi(t)$ is the Lagrange multiplier. The optimal laser pulse $\boldsymbol{\epsilon}(t)$ is provided at the end of the iterative procedure ${ }^{13}$ We point out that the initial pulse (zeroth iteration) is sinusoidal and has both $x$ and $y$ components, whereas the converged optimal pulse is always found to be polarized in the $x$ direction, i.e., $\boldsymbol{\epsilon}(t)=\epsilon(t) \hat{x}$. In Eq. (7), we choose a sinusoidal pulse envelope $A(t)=\sin ^{2}(\pi t / T)$ and restrict the pulse intensity by a penalty factor $\alpha$. Unless stated otherwise, we have fixed $\alpha=0.5$ leading to pulse intensities $10^{3}, \ldots, 10^{4} \mathrm{~W} / \mathrm{cm}^{2}$. We apply a rapidly converging numerical scheme ${ }^{13,14}$ that has been implemented in the OCTOPUS code. ${ }^{15}$

We consider excitations only from the ground state and set $\left|\Phi_{\mathrm{I}}\right\rangle=|00\rangle$. Figure 2 shows the maximum overlaps $\left|\left\langle\Psi(T) \mid \Phi_{\mathrm{F}}\right\rangle\right|^{2}$, i.e., maximum occupations of the target states $|10\rangle$ and $|20\rangle$ as a function of the interdot distance (note the logarithmic scale). The pulse lengths are fixed to $T=50$ and $T=100$, respectively. When $d$ is increased, the target-state occupations increase from the HO value of $\lesssim 0.6$ (see Ref. 10) exponentially to close to one. As expected, at large interdot distances $(d \geqslant 8)$, corresponding to the uncoupling of the DQD, the occupations for $|00\rangle \rightarrow|20\rangle$ decrease back to the $\mathrm{HO}$ value marked by a dashed line. On the other hand, the occupation for $|00\rangle \rightarrow|10\rangle$ increases even further in this limit due to the asymptotic degeneracy of the states (see above). Generally, for this transition, a pulse length of $T=50$ a.u. $\sim 3 \mathrm{ps}$ is sufficient to achieve high occupations. In the transition $|00\rangle \rightarrow|20\rangle$ instead, $T=100$ a.u. $\sim 6 \mathrm{ps}$ is needed for the same accuracy.

As seen in Fig. 2, the target-state occupations above 90\% are generally obtained at distances $2<d<8$, i.e., at $d \sim 20, \ldots, 80 \mathrm{~nm}$. This length scale is well realizable in experiments. ${ }^{1-3}$ We point out that the most distinctive devia- 

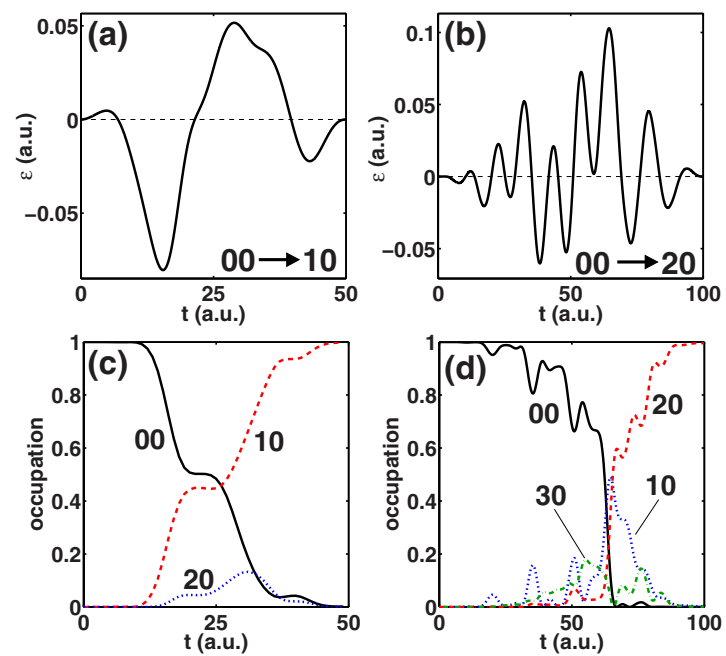

FIG. 3. (Color online) Upper panel: optimized pulses ( $x$ components) for transitions (a) $|00\rangle \rightarrow|10\rangle$ and (b) $|00\rangle \rightarrow|20\rangle$. The interdot distances are fixed to $d=3$ and 5 and the pulse lengths to $T$ $=50$ and 100, respectively. Lower panel: occupations of states involved in the transitions.

tions in the occupations in this regime arise from resonance effects. For example, the jump in the $|00\rangle \rightarrow|10\rangle$ transition at $d=3.6, \ldots, 3.7$ (see the arrow in Fig. 2), where the occupation decreases from 0.9997 to 0.9591 , is due to the degeneracy of $\omega_{00}^{10}$ and $\omega_{10}^{20}-\omega_{20}^{30}$. The degeneracy disturbs the optimal transition path leading to reduced maximum occupation.

In Fig. 3, we give two examples of optimized pulses and the occupations of states during transitions: [(a) and (c)] $|00\rangle \rightarrow|10\rangle$ when $d=3$ and [(b) and (d)] $|00\rangle \rightarrow|20\rangle$ when $d=5$. The pulse for $|00\rangle \rightarrow|10\rangle$ has the resonant frequency $\omega_{00}^{10}$ as the major component, but as seen in the occupations, state $|20\rangle$ is also populated during the transition. For comparison, we exposed the system also to a cw having the resonant (Rabi) frequency $\Omega_{R}=\omega_{00}^{10}$ and the same fixed length $(T=50)$ as the optimized pulse. We set the pulse amplitude to $\Omega_{R} / \mu_{00}^{10}=\pi /\left(\mu_{00}^{10} T\right)$ to satisfy the $\pi$-pulse condition. This leads to occupation of 0.93 of state $|10\rangle$. In view of the 0.999 occupation achieved by the optimal pulse, OCT is superior to the cw approach. A comprehensive comparison of occupations achieved with different pulse lengths applying OCT and cw's for a quantum-ring system is given in Ref. 12 .

As shown in Fig. 3(d), the $|00\rangle \rightarrow|20\rangle$ transition must be mediated by the $|10\rangle$ state, since a direct transition is forbidden by the dipole selection rules. However, the intermediate state $|10\rangle$ does not need to get fully populated during the optimized transition process. The higher states are also involved in the process, and in this example, the state $|30\rangle$ receives considerable occupation with a maximum of $\sim 20 \%$ at $t \sim 90$. The final target-state occupation is 0.998 , whereas using two resonant cw's one after the other, we could not exceed 0.9 (pulse length fixed to $T=100$ ). Generally, in multilevel transitions of this type, the efficiency of OCT is pronounced with respect to cw's due to the multiplication of errors in the latter approach when full population of intermediate states is required before changing the resonant frequency.
The control of electron transport in the DQD requires initialization of the state by localizing the electron in the one of the dots. Namely, the single-electron ground state of the DQD is a gerade state with half of the electron in one well and half in the other (see the lowermost figure in the right panel of Fig. 1). Only in the limit $d \rightarrow \infty$, the localized states become degenerate eigenstates. However, the initial state can be fixed with certainty by applying a constant external field or by adjusting the gates in the DQD device in order to create a potential shift between the two dots. After the initial localization, electron transport into another well can be driven using a linear switch or a cw with the resonant confinement frequency $\omega_{0}$. These types of transport on time scales of $10^{-10} \mathrm{~s}$, close to the required times for SWAP operations in experiments, ${ }^{1}$ were reported very recently by Førre et $a l .{ }^{4}$

Now, we show that OCT provides a very fast (switching times of a few picoseconds) and stable alternative to control the electron transport in a DQD. First, we break the degeneracy of the ground state by setting the external potential in the (lower) left dot $V_{c}^{\text {left }} \rightarrow V_{c}^{\text {left }}-0.2$. Then, the ground state $\left|00_{L}\right\rangle=|L\rangle$ and the first excited state $\left|00_{R}\right\rangle=|R\rangle$ correspond to electron localization in the (lower) left and (upper) right dots, respectively, provided that $d$ is sufficiently large. The result of the OCT calculation for transition $|L\rangle \rightarrow|R\rangle$ is shown in Fig. 4. The pulse length is fixed to $T=100$ ( $\sim 6 \mathrm{ps}$ ), and the interdot distance $d=6$ corresponding to relatively large coupling between the dots. The spectrum of the optimized pulse (a) has a large peak at the resonant frequency $\omega=\omega_{0}=0.5$ a.u. $\sim 9 \mathrm{THz}$ and a few smaller peaks at $\omega \sim 0.2, \ldots, 0.3$ and $\omega \sim 1$. The small peaks correspond to transitions in the higher states which get significantly populated in the transitions. This is visualized in Fig. 4(b) showing the occupations of $|L\rangle$ and $|R\rangle$ (solid lines) that sum up only to about $50 \%$ in the middle of the transition at $T \sim 50$. We also plot the integrated electron densities $\rho_{R}$ and $\rho_{L}$ (dashed lines) in the (lower) left and (upper) right parts of the DQD, respectively. The quasiperiodic oscillations in the densities indicate that the electron charge transfers in blobs as visualized in the snapshots in Figs. 4(c)-4(h). In this example, we find the final occupation $|\langle\Psi(T) \mid R\rangle|^{2}=0.985$. It is worth noting that the final occupations are closer to one at larger interdot distances so that the coupling between the dots is weaker. In this regime, the optimized pulses may attain a linear slope. This immediately suggests that a combination of a linear field and OCT could be ideal in controlling transport in the uncoupled regime. In this work, however, we focus on coupled DQDs which we, in fact, find the most challenging in terms of electron control in nanoscale applications.

There are three significant advantages in OCT with respect to a linear switch or a resonant $\mathrm{cw}$ when generating electron transport in DQDs. First, the optimization procedure is insensitive to the interdot coupling. The cw approach, in principle, requires an uncoupled system for electron transport with unit probability. ${ }^{4}$ We tested a cw with the resonant frequency $\omega_{0}$ for the above example with various amplitudes and could not exceed occupation of 0.4 of the target state $|R\rangle$. Second, the optimized pulses can be very short in order to achieve sufficient occupation, since the higher-lying states are incorporated in the control problem. In the above ex- 

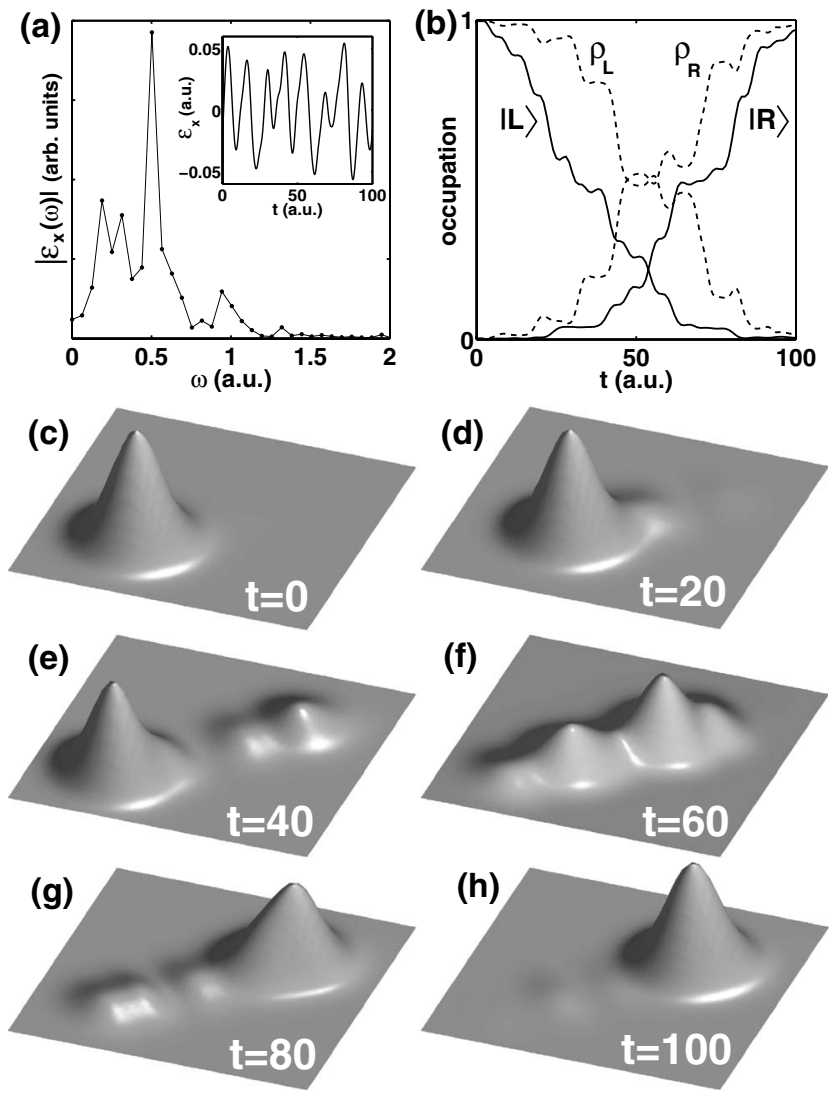

FIG. 4. (a) Spectrum of the optimized pulse (inset) for the electron transport process $|L\rangle \rightarrow|R\rangle$ in a fixed time $T=100$. The pulse has a rectangular envelope $A(t)=1$, and the penalty factor is $\alpha=1$. (b) Occupations of states $|R\rangle$ and $|L\rangle$ (solid lines) and the integrated densities $\rho_{R}$ and $\rho_{L}$ (dashed lines) in the right and left dots during the transport. (c)-(h) Snapshots of the total electron density $\rho_{R}$ $+\rho_{L}$. The double quantum dot has $d=6, \omega_{0}=0.5$, and well-depth asymmetry of $V_{0}=0.2$.

ample the pulse length of $\sim 6$ ps is more than by a factor of 10 smaller than in linear and cw switches and well below the recently measured coherence times of hundreds of nanoseconds in DQDs. ${ }^{3}$ Third, and probably most important, the OCT approach is insensitive to deviations in the external potential, since the specific shape of the DQD is taken into account explicitly through the external potential in the Hamiltonian. We tested this by adding a fourth-order anharmonic term in the external potential [Eq. (1)]. This results in a change in the optimal pulse shape and in an increase in the required pulse length (in the case of a positive anharmonicity). However, we found no decrease in the obtained targetstate occupation when using anharmonicities that lead to dramatic loss of accuracy in the cw approach. ${ }^{4}$ In practical applications, the exact shape of the external potential could be obtained by measuring the differential conductance in a single-electron transport experiment and thereafter numerically solving the (inverse) Schrödinger equation. This approach has been applied to modeling external impurities inside single quantum dots. ${ }^{16}$

We expect that the experimental creation of optimal laser pulses, such as presented in this work, will be soon within reach of laser technologies. The intensities of $10^{3}-10^{4} \mathrm{~W} / \mathrm{cm}^{2}$ required for DQD excitations can be already obtained in the terahertz regime by high-power freeelectron lasers. ${ }^{17}$ On the other hand, shaping of picosecond terahertz pulses has been recently made possible by employing transient polarization grating. ${ }^{18}$ Quantum cascade lasers ${ }^{19}$ may also provide an applicable route for precise pulse shaping in the terahertz regime. The rapid developments in the terahertz laser technology ${ }^{20}$ will eventually lead to the combination of sufficient pulse power and accurate manipulation of the pulse shape.

Finally, we point out that in our future work, we aim at combining our approach with magnetic-field optimization which could allow us to coherently control the spin state simultaneously with the electron localization.

This work was supported by the EU's Sixth Framework Programme through the Nanoquanta NoE (No. NMP4-CT2004-500198), SANES project (No. NMP4-CT-2006017310), DNA-Nanodevices (No. IST-2006-029192), Barcelona Supercomputing Center, the Humboldt Foundation, the Academy of Finland, the Finnish Academy of Science and Letters through the Viljo, Yrjö and Kalle Väisälä Foundation, and the Deutsche Forschungsgemeinschaft (No. SFB 658).

*esa@physik.fu-berlin.de

${ }^{1}$ J. R. Petta, A. C. Johnson, J. M. Taylor, E. A. Laird, A. Yacoby, M. D. Lukin, C. M. Marcus, M. P. Hanson, and A. C. Gossard, Science 309, 2180 (2005).

${ }^{2}$ J. R. Petta, A. C. Johnson, C. M. Marcus, M. P. Hanson, and A. C. Gossard, Phys. Rev. Lett. 93, 186802 (2004).

${ }^{3}$ J. Gorman, D. G. Hasko, and D. A. Williams, Phys. Rev. Lett. 95, 090502 (2005).

${ }^{4}$ M. Førre, J. P. Hansen, V. Popsueva, and A. Dubois, Phys. Rev. B 74, 165304 (2006).

${ }^{5}$ I. Grigorenko, O. Speer, and M. E. Garcia, Phys. Rev. B 65, 235309 (2002).

${ }^{6}$ S. G. Kosionis, A. F. Terzis, and E. Paspalakis, Phys. Rev. B 75,

193305 (2007)

${ }^{7}$ A. Harju, S. Siljamäki, and R. M. Nieminen, Phys. Rev. Lett. 88, 226804 (2002).

${ }^{8}$ A. Wensauer, O. Steffens, M. Suhrke, and U. Rössler, Phys. Rev. B 62, 2605 (2000).

${ }^{9}$ A. P. Peirce, M. A. Dahleh, and H. Rabitz, Phys. Rev. A 37, 4950 (1988); R. Kosloff, S. A. Rice, P. Gaspard, S. Tersigni, and D. J. Tannor, Chem. Phys. 139, 201 (1989).

${ }^{10}$ See, e.g., A. G. Butkovskiy and Y. I. Samoilenko, Control of Quantum-Mechanical Processes and Systems (Kluwer, Dordrecht, 1990).

${ }^{11}$ S. G. Schirmer, H. Fu, and A. I. Solomon, Phys. Rev. A 63, 063410 (2001). 
${ }^{12}$ E. Räsänen, A. Castro, J. Werschnik, A. Rubio, and E. K. U. Gross, Phys. Rev. Lett. 98, 157404 (2007).

${ }^{13}$ W. Zhu and H. Rabitz, J. Chem. Phys. 109, 385 (1998).

${ }^{14}$ J. Werschnik, Quantum Optimal Control Theory: Filter Techniques, Time-Dependent Targets, and Time-Dependent DensityFunctional Theory (Cuvillier, Göttingen, 2006).

${ }^{15}$ A. Castro, H. Appel, M. Oliveira, C. A. Rozzi, X. Andrade, F. Lorenzen, M. A. L. Marques, E. K. U. Gross, and A. Rubio, Phys. Status Solidi B 243, 2465 (2006).
${ }^{16}$ E. Räsänen, J. Könemann, R. J. Haug, M. J. Puska, and R. M. Nieminen, Phys. Rev. B 70, 115308 (2004).

${ }^{17}$ G. W. Williams, Rep. Prog. Phys. 69, 301 (2006).

${ }^{18}$ A. G. Stepanov, J. Hebling, and J. Kuhl, Opt. Express 12, 4650 (2004).

${ }^{19}$ M. Tonouchi, Nat. Photonics 1, 97 (2007).

${ }^{20}$ For reviews, see, e.g., J. H. Posthumus, Rep. Prog. Phys. 67, 623 (2004); K. Reimann, ibid. 70, 1597 (2007). 